\title{
Heparin Coating on Ureteral Double J Stents Prevents Encrustations: An in Vivo Case Study
}

\author{
FURIO CAUDA, M.D., ${ }^{2}$ VALENTINA CAUDA, Ph.D., ${ }^{1}$ CRISTIAN FIORI, M.D., ${ }^{2}$ \\ BARBARA ONIDA, Prof., ${ }^{1}$ and EDOARDO GARRONE, Prof. ${ }^{1}$
}

\begin{abstract}
Purpose: To evaluate the ability of heparin coating to inhibit Double $\mathbf{J}$ stent encrustation and compare it with the classic polyurethane Double J stent.

Patients and Methods: The study involved five patients with bilateral obstructions, who required bilateral ureteral Double J stent placement. Every patient received a heparin-coated Double J stent and a traditional polyurethane Double J stent for 1 month. After removal, the stents were analyzed using field emission scanning electron microscopy (FESEM), energy dispersive spectroscopy (EDS). and micro-infrared spectrophotometry (Micro-IR). These same techniques were used to analyze the heparin-coated and uncoated stents before insertion. The thickness, extension, and composition of encrustation of the coated and uncoated stents were compared. Moreover, two heparin-coated stents were analyzed with the same techniques after they had been in place for 10 and 12 months.

Results: FESEM analysis showed that the difference in encrustation thickness and extension between the two groups was significant. EDS and Micro-IR confirmed that in the heparinized stents the encrustations were not as uniform and compact as those in the uncoated stents. The stents that were left in place long-term were free of encrustations and had no changes in the heparin layer.

Conclusions: Heparin coating reduces stent encrustation. Moreover, as no changes were seen in the heparin layer, we concluded that covalent heparin bonding enhances its adhesion to the polyurethane surface and ensures its stability for long periods. The heparin-coated stent appears to be a useful tool for long-term urinary drainage.
\end{abstract}

\section{INTRODUCTION}

$\mathbf{U}$ RETERAL STENTING FOR URINARY DRAINAGE HAS BECOME A ROUTINE PROCEDURE IN UROLOGY. The majority of stents are used temporarily, particularly in stone-forming patients. Sometimes the stent may also be a permanent solution, especially in patients with malignant ureteral obstruction; in these cases, encrustations are a major problem, both in terms of patient quality of life and in terms of economic costs. ${ }^{1,2}$

The initial step in encrustation of any urinary drainage device appears to be bacterial colonization and the formation of a layer of microorganisms that accumulates on the surface, along with their by-products. ${ }^{1,3,4,5}$ The presence of this layer, called "biofilm," in combination with elevation of urinary $\mathrm{pH}$ and changes in electrolyte composition, is responsible for crys- tal formation and stent encrustation. ${ }^{3-5}$ The development of encrustations can cause stent obstruction with impaired urine flow, which can compromise patient care and may lead to pyelonephritis, sepsis, and shock. ${ }^{6}$

Various surface modifications to medical devices have been developed to prevent bacterial adhesion, such as silver-coated surfaces, controlled-release antibiotics, and surface modifications to change hydrophobicity or functional groups having antimicrobial activity. ${ }^{7-11}$

Heparin coating was proposed to prevent bacterial adhesion over the last three decades, especially in vascular medicine. ${ }^{12-14}$ Heparin is an anticoagulant that carries a strong negative electrical charge and helps prevent cell adhesion. For this reason, heparinization can be a practical and low-cost approach to the prevention of catheter-associated bacteremia or fungemia. ${ }^{11,15}$

${ }^{1}$ Dipartimento di Scienza dei Materiali e Ingegneria Chimica, Politecnico di Torino, and ${ }^{2}$ Dipartimento di Nefrourologia , S.S.C.V.D. per il trattamento integrato della calcolosi urinaria, Ospedale Maggiore S. Giovanni Battista, Torino, Italy. 
Table 1. Clinical Characteristics of the Patients in This Study

\begin{tabular}{|c|c|c|c|c|}
\hline Patient no. & Gender & Age (y) & Stenting indication: Right side & Stenting indication: Left side \\
\hline 1 & M & 50 & $\begin{array}{l}\text { Post-ureteroscopic procedure to } \\
\text { remove a ureteral stone } \\
\text { (heparin-coated) }\end{array}$ & $\begin{array}{l}\text { Post-ureteroscopic procedure to remove } \\
\text { ureteral stone (uncoated polyurethane) }\end{array}$ \\
\hline 2 & $\mathrm{~F}$ & 55 & $\begin{array}{l}\text { Hydronephrosis due to UPJ } \\
\text { obstruction }^{\text {a }} \text { (polyurethane) }\end{array}$ & $\begin{array}{l}\text { Post-ureteroscopic procedure to remove } \\
\text { ureteral stone (heparin-coated) }\end{array}$ \\
\hline 3 & $\mathrm{~F}$ & 56 & $\begin{array}{l}\text { Hydronephrosis due to UPJ } \\
\text { obstruction }^{\text {a }} \text { (heparin-coated) }\end{array}$ & $\begin{array}{l}\text { Hydronephrosis due to UPJ obstruction } \\
\text { (uncoated polyurethane) }\end{array}$ \\
\hline 4 & M & 54 & $\begin{array}{l}\text { Post-flexible ureteroscopic } \\
\text { procedure for ureteral stone } \\
\text { and upper pole kidney stone } \\
\text { (uncoated polyurethane) }\end{array}$ & $\begin{array}{l}\text { Post-ureteroscopic procedure for ureteral } \\
\text { stone (heparin-coated) }\end{array}$ \\
\hline 5 & M & 51 & $\begin{array}{l}\text { Post-flexible ureteroscopic } \\
\text { procedure for ureteral stone } \\
\text { and lower pole kidney stone } \\
\text { (uncoated polyurethane) }\end{array}$ & $\begin{array}{l}\text { Post-ureteroscopic procedure to remove } \\
\text { ureteral stone (heparin-coated) }\end{array}$ \\
\hline
\end{tabular}

${ }^{a}$ After stent removal the patient was treated with pyeloplasty.

Here we report on encrustation of heparin-coated Double J stents in comparison to the classic polyurethane stent.

\section{PATIENTS AND METHODS}

This study involved patients with bilateral ureteral obstructions who required bilateral placement of Double J stents. Patients with urinary stones or residual stone fragments remaining after endoscopic procedures were excluded. Five patients matched our inclusion criteria, and clinical data were recorded such as age, gender, and indications for stent placement (Table 1). Patients gave informed consent for the study.

A randomly chosen heparin-coated Double J stent (6F, 26 $\mathrm{cm}$ ) (Endosof-Radiance, Cook Medical, Bloomington, IN) was placed in one ureter, and a traditional uncoated polyurethane Double J stent $(6 \mathrm{~F}, 26 \mathrm{~cm})$ was inserted in the other ureter. The stents were placed during cystoscopy, and in all patients a retrograde uretero-pyelogram was performed at the start of the procedure to evaluate the excretory system. Ciprofloxacin (500 $\mathrm{mg}$ twice a day) was given for prophylaxis on days $0,1,2$ and 3 post-procedure. In all patients the stents were removed after 1 month with another cystoscopy. These parameters were recorded: occurrence of technical problems, violations of asep- tic technique during the procedures, antibiotic therapy, other therapeutic interventions performed while the stents were indwelling, and the presence of fever, infection, or urinary symptoms. Urinalysis and cultures were performed on day 15 postprocedure.

After removal, the stents were randomly cut into small sections both longitudinally and transversely for study. For the longitudinal sections, both the inner and outer surfaces were analyzed, while in the transverse sections one cut surface per section was studied. The two edges of the stents were also studied in transverse section.

Morphologic and compositional analyses were carried out. These analyses were carried out by field emission scanning electron microscopy (Assing FESEM Supra 25; Gottingen, Germany) and energy dispersive spectroscopy (EDS) (INCA XSight; Oxford Instrument, Gottingen, Germany). To perform these tests, the stents had to be covered by a thin layer of gold to become conductive. The thickness of stent encrustation was measured on the transverse sections using INCA $^{\circledR}$ electronic imaging software during the FESEM testing; if encrustation was present but not measurable, the thickness was considered as zero.

As the encrustation thickness was variable, measurements were carried out at different points, and the highest thickness

Table 2. Clinical Characteristics of Two Patients Who Had Coated Stents in Place for 10 and 12 Months

\begin{tabular}{|c|c|c|c|}
\hline Case no. & Gender & Age (y) & Clinical data and stenting indications \\
\hline 6 & $\mathrm{~F}$ & 45 & $\begin{array}{l}\text { Polycystic disease of the kidneys, liver, and ovaries, with severe kidney } \\
\text { failure; hydronephrosis on the right side due to extrinsic compression; she } \\
\text { had no surgical indications, and thus was treated with ureteral placement of a } \\
\text { Double J stent; the patient had previously experienced repeated obstructions } \\
\text { of an uncoated polyurethane stent }\end{array}$ \\
\hline 7 & $\mathrm{~F}$ & 74 & $\begin{array}{l}\text { Hydronephrosis due to UPJ obstruction on the right side; the patient was } \\
\text { previously treated with open pyeloplasty and endopyelotomy without no } \\
\text { effect; she then refused another intervention, and was treated with placement } \\
\text { of a ureteral Double J stent; the patient had previously experienced repeated } \\
\text { obstructions of an uncoated polyurethane stent }\end{array}$ \\
\hline
\end{tabular}




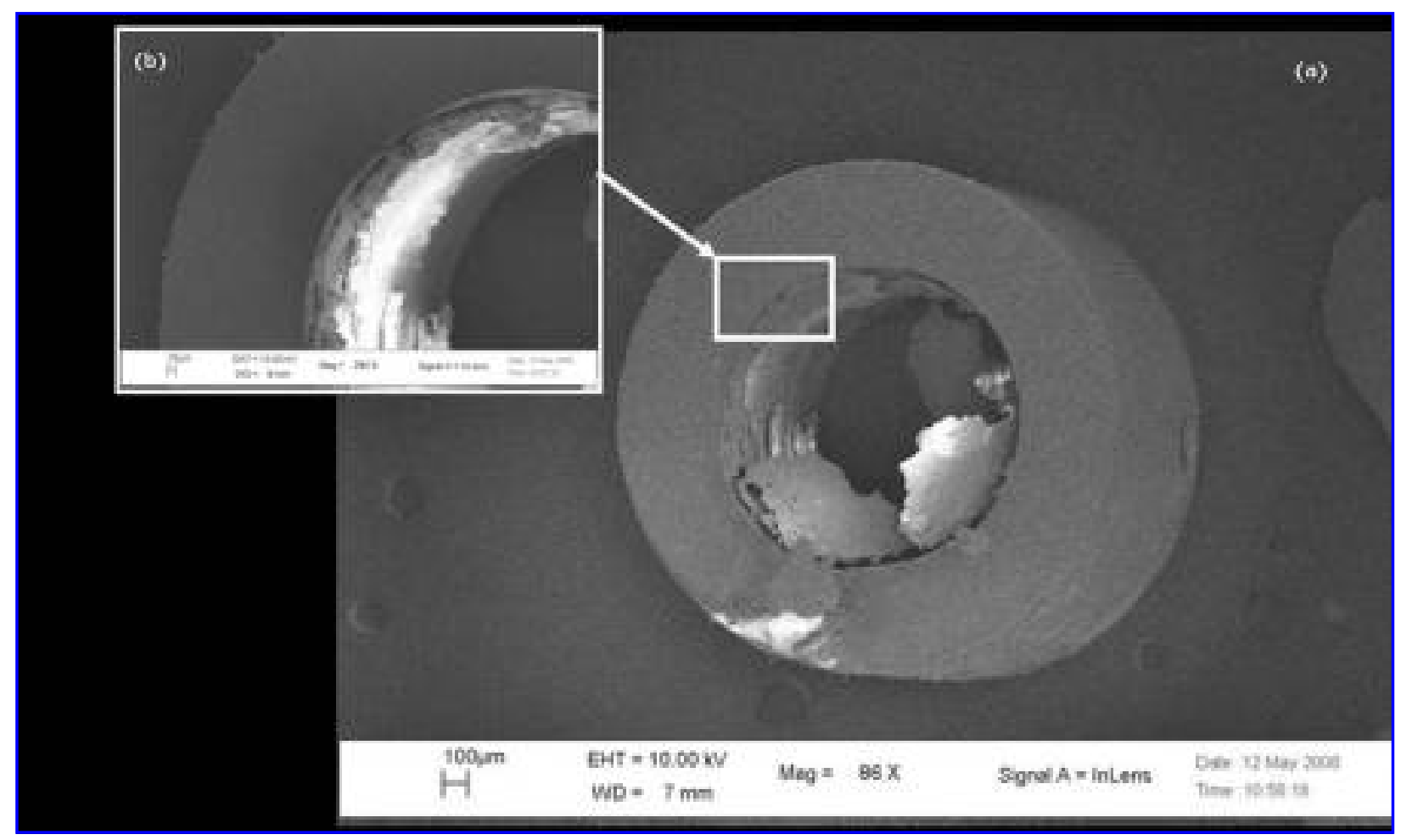

FIG. 1. (a) Cross-section of an uncoated stent. FESEM analysis showed an irregular inner surface due to encrustations. (b) Close-up of the cross-section. The encrustation is clearly visible, and its thickness was $20 \mu \mathrm{m}$.

value registered per transverse section was used for statistical analysis.

For each longitudinal section, encrustation was measured as a percentage of coverage of the stent's surface area, and a PC was used for image analysis.

Statistical analyses to evaluate the differences between the two groups (coated $v$ uncoated stents) were carried out using Student's $t$-test, chi-square test, and Fisher's exact test. All data were analyzed using software (Statistica; StatSoft Inc., Tulsa, OK) run on a PC.

Compositional studies were carried out with a micro-infrared spectrophotometer (Micro-IR) (Bruker Optik; Ettingen, Germany) in the attenuated total reflection (ATR) mode. This instrument facilitates inspection of the longitudinally cut sample surface with an optical microscope, and then analysis of its composition through the IR spectrophotometer. This allowed us to study both the inner and outer surfaces of the stents.

For comparison purposes, these same techniques were used to analyze both types of stents before insertion.

In addition to the five patients described above, two other patients with chronic ureteral obstruction were involved. Previously, both patients had ureteral stenting with polyurethane Double J stents and experienced repeated stent obstructions. Their clinical data were recorded (Table 2), and a heparincoated stent (Endosof-Radiance) was placed and removed after they had been in place for 10 and 12 months. After stent removal, morphologic and compositional analyses were performed as described above.

\section{RESULTS}

\section{Clinical and procedural data}

No technical problems or violations of asepsis were recorded during the endoscopic procedures. In all patients retrograde uretero-pyelography performed at the start of the procedure showed bilateral ureteral obstruction with various degrees of excretory system dilatation.

During the study period no patient reported fever or flank pain. Urine culture was negative in all patient. One patient had symptoms of frequency and urgency, and was effectively treated with antimuscarinics until stent removal. Stents were easily removed after one 1 month in all cases.

No technical problems occurred during the endoscopic procedures on the two patients with chronic unilateral obstruction. In these patients uretero-pyelography showed ureteral obstruction with severe excretory system dilatation. During the study period, no fever, flank pain, or urinary symptoms were recorded, and urine cultures were negative in both patients.

\section{Stent analysis}

Pre-insertion stent analysis. The polyurethane uncoated stents were characterized by a largely regular surface, with a few irregularly-shaped particles (polyurethane or impurities) about 0.5 to $1 \mu \mathrm{m}$ in size. In the coated stents, the heparin coating can clearly be seen, its thickness being about $5 \mu \mathrm{m}$. The elemental analysis at $10 \mathrm{kV}$ detects only the heparin coating and 


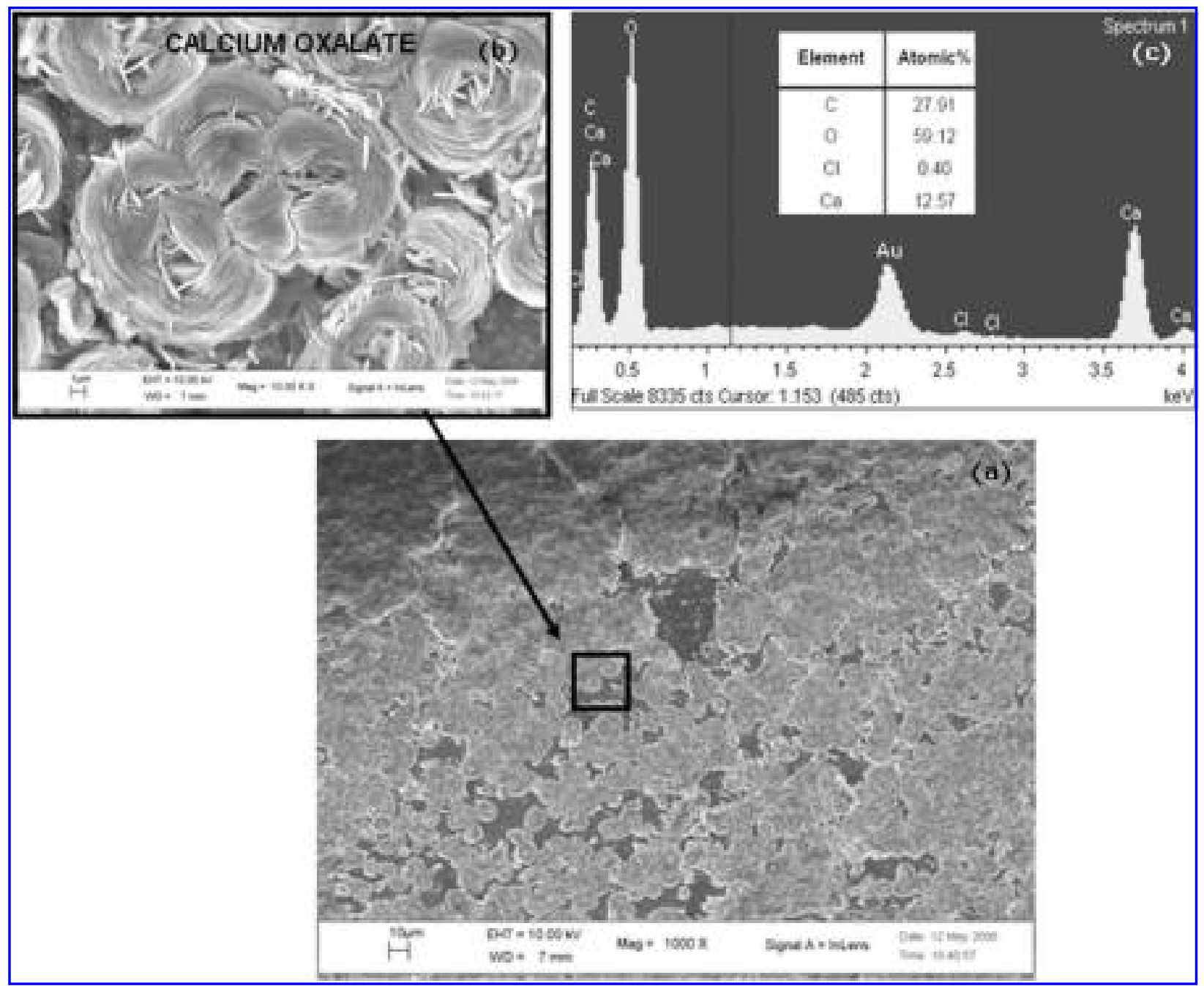

FIG. 2. (a) FESEM image showing crystals of calcium oxalate on the surface of an uncoated stent. (b) Higher-magnification EDS image demonstrating the presence of calcium oxalate.

not the polyurethane substrate, since the coating was thicker than the depth of the analysis possible (approximately $1 \mu \mathrm{m}$ at that voltage). Barium was also present to make them visible on X-rays.

Stents post-removal after 1 month. Overall, two types of deposits were detected on the uncoated stents: (1) amorphous, crystalline inorganic deposits; and (2) bacterial biofilm. These stents showed quite severe encrustations on both on the inner and outer surfaces (Fig. 1). Encrustations were identified and measured on 26 of the 34 total cut surfaces. Upon FESEM analysis of the transverse sections, the average encrustation thickness on the uncoated stents was $17.0 \mu \mathrm{m}( \pm 12.1 \mu \mathrm{m})$. The average encrustation coverage on the surfaces of the longitudinal sections was $86 \%$ ( $\pm 31 \%)$, as measured on both the inner and outer surfaces.

EDS analysis showed that the inorganic deposits were made up of oxygenated calcium compounds, along with magnesium, potassium, and sodium chloride. The EDS also revealed the presence of carbon, likely due to bacterial deposits in the biofilm, rather than from the polyurethane itself, as the depth of analysis possible at $10 \mathrm{kV}$ is shallower than the thickness of the biofilm. Some crystals with regular geometry were seen on the outer surfaces of the uncoated stents (Fig. 2). Elemental analysis showed they were calcium oxalate crystals.

The Micro-IR spectra of the uncoated stents after removal were collected from both the internal and external surfaces of the longitudinal sections and compared with the spectra of the stents before insertion.

Figure 3 shows spectra of an uncoated stent, confirming the presence of two kinds of encrustation on the internal stent surface [spectra (a) and (b)]. Spectrum (a) is attributable to the bacteria 1 biofilm, and spectrum (b) shows peaks ascribable to calcium oxalate, results that agreed with those of the EDS analysis. After stent removal both spectra were quite different from the control analyses done before insertion [spectrum (c)]. In particular, the peaks characteristic of polyurethane are no longer seen in spectra (a) and (b). The spectra of all longitudinal sections (both the inner and outer surfaces) were similar. 


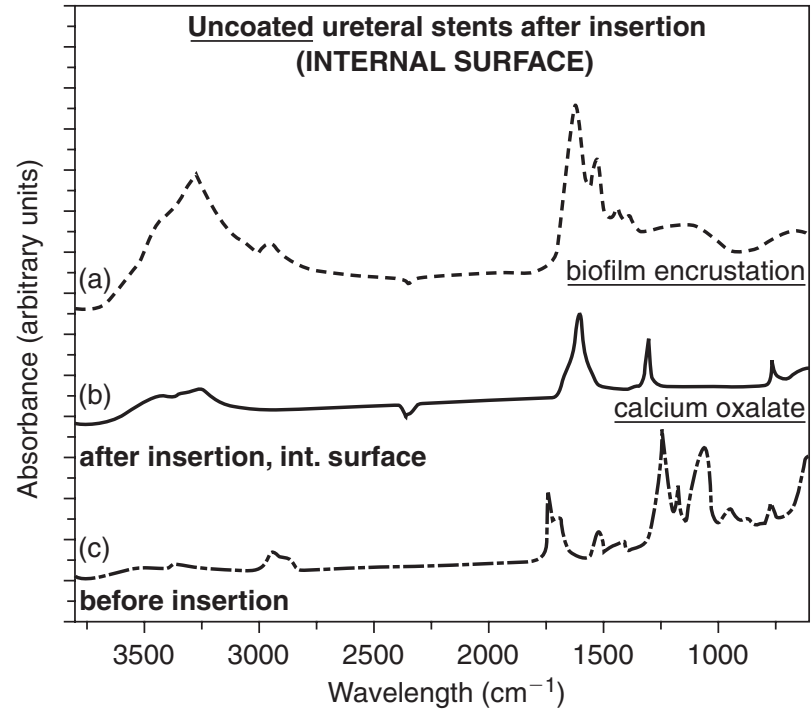

FIG. 3. Micro-IR analysis of the inner surface of an uncoated stent. Three spectra are shown: (a) a typical spectrum of organic biofilm, and (b) a spectrum of inorganic encrustation. The presence of calcium oxalate was suspected, and EDS analysis (not shown here) confirmed this fact. (c) Spectrum of polyurethane. Note that the peaks characteristic of polyurethane are no longer seen in spectra (a) and (b) (see text for details).

A different situation was seen for the heparin-coated stents. Overall, FESEM analysis showed less encrustation both on their internal and external surfaces than those of the uncoated stents. The heparin was always still visible at the cut edge of the stent (Fig. 4). In some cases, a layer of encrustation was present on the catheter's surface, though it was thinner than the deposits seen on the uncoated stents. Encrustations were identified and measured on 20 of 44 total cut surfaces. FESEM analysis of the transverse sections of the uncoated stents showed that the average encrustation thickness was $8.5 \mu \mathrm{m}( \pm 8.6 \mu \mathrm{m})$, and the average extent of encrustation on the longitudinal sections was
$67 \%( \pm 33 \%)$, as measured both on the inner and outer surfaces.

The elemental EDS analysis confirmed the presence of heparin in the layer seen at the cut edges of the coated stents. Furthermore, EDS showed that the encrustations found on FESEM analysis were composed of calcium oxalate.

After removal, the Micro-IR spectra of the coated stents were collected for both the internal and external surfaces of the longitudinal sections, and compared with the spectra of the stents before insertion.

Overall, the spectra of the coated stents were different from those of the uncoated stents, particularly in the areas free of encrustations. Spectrum (e) in Figure 5, from a unencrusted area of a coated stent, is quite similar to spectrum (f), which is from a heparinized surface before insertion. In contrast, spectrum (d), collected from an encrusted area of the inner surface, is different from that of the heparin-coated stent before insertion, verifying the presence of organic bacterial encrustation, the thickness of which could not be measured using Micro-IR. Although there was some biofilm present on the coated catheters, it was not as uniform and compact as were the encrustations on the uncoated stents. Overall, the spectra of the external surfaces of the coated stents were quite similar to those of the stents before insertion, confirming the findings of FESEM.

Statistical analysis was performed on the FESEM results. The difference in extent of encrustation seen in the two groups was statistically significant (26 of 34 cut surfaces for the uncoated stents $v 20$ of 44 cut surfaces for the coated stents; $P=$ $<0.005)$.

Moreover, a statistically significant difference was found, both for encrustation thickness (on transverse sections; $17.0 \mu \mathrm{m}$ $v 8.5 \mu \mathrm{m}$, for the uncoated and coated stents, respectively; $P<$ 0.05 ) and for extent of encrustation (on longitudinal sections; $86 \%$ v $67 \%$, for the uncoated and coated stents, respectively; $P<0.05)$.

Stents post-removal after 10 months. The coated stent did not show any encrustation or degradation of the heparin sur-

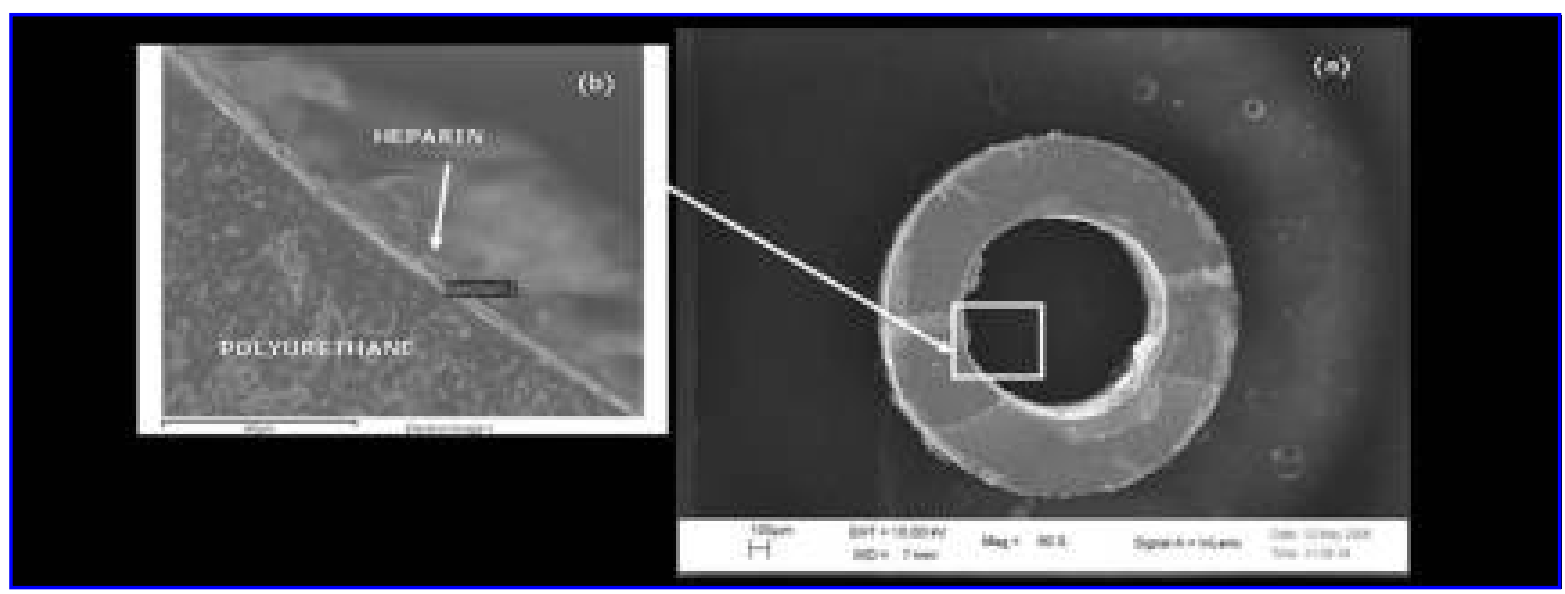

FIG. 4. (a) Cross-section of a heparin-coated stent. FESEM analysis showed a largely smooth surface with some encrustation. Note that the thickness and extent of these encrustations are quite different from those seen on an uncoated stent (see Fig. 1; both these stents were from the same patient). (b) Magnification of encrustation at the stent's edge. This FESEM image clearly shows the presence of a thin layer over the polyurethane surface. EDS analysis (not shown here) confirmed that it was heparin. 


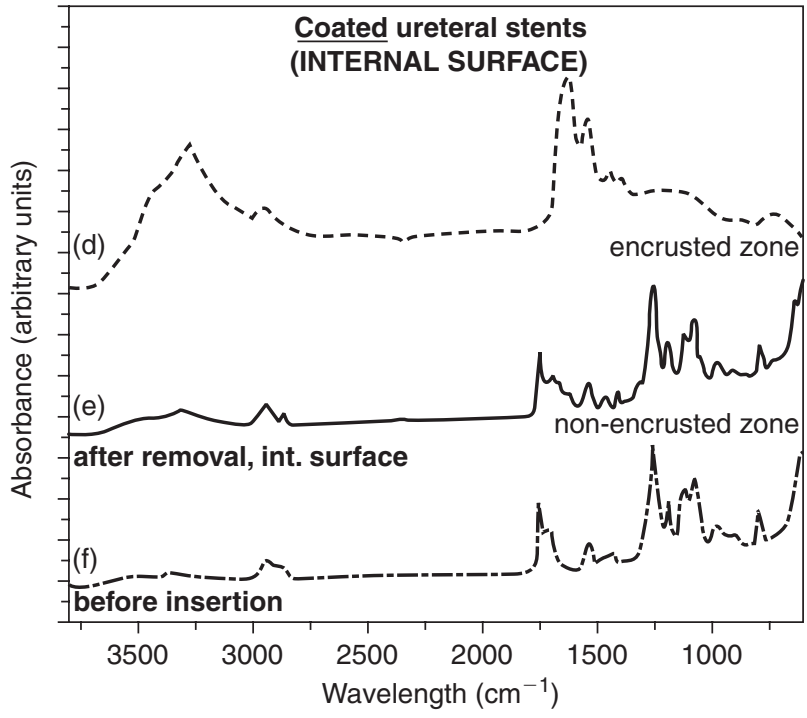

FIG. 5. (d and e) Micro-IR spectra from the internal surface of the coated stents, showing areas with different levels of encrustation. Spectrum (e) is quite similar to the spectrum (f) of the heparinized surface before insertion, and reveals an absence of encrustations.

face. The cross-section of the catheter was completely free of biofilm, as were the drainage pores on the outer surface (Fig. 6). Elemental analysis was carried out on the internal surface, which is usually the most encrusted part of the stent. Small grains were observed; EDS analysis, however, showed the presence of carbon and oxygen, indicating that these particles were made of polyurethane.

Micro-IR spectra confirmed the findings obtained by electron microscopy and elemental analysis. No encrustations were identified, and there were no significant changes in the heparin layer after the stent was in place for 10 months.

Stents post-removal after 12 months. After being in place for 1 year, the coated stent was visibly free of encrustations (Fig. 7a), as confirmed by electron microscopy and EDS analysis. However, the outer surface had a bacterial biofilm containing sodium chloride and oxygenated calcium compounds (Fig. 7b).
Heparin was seen through fractures in the bacterial biofilm, but the heparin layer itself was not affected. No encrustations or biofilm was found on the internal surface of the stent. Micro-IR spectra (Fig. 8) collected both at the internal and external surfaces of the coated stent supported this finding, as the spectrum of the outer surface showed evidence of a bacterial biofilm. The inner surface appeared free of encrustation and the spectrum showed peaks of heparin and polyurethane of lower intensity, likely due to the deposition of an undetectable amount of encrustation. As the peaks are very similar to those seen in the spectrum before insertion, it appears there were no significant modifications of the heparin or polyethylene after being in place for 1 year.

\section{DISCUSSION}

Encrustation and subsequent obstruction of ureteral Double J stents or nephrostomy tubes pose significant medical and economic problems. ${ }^{1,2,5,16}$ Many strategies have been developed to prevent encrustation, but thus far none has proved to be optimal. A silver coating showed high antibacterial activity in vitro but was ineffective in vivo ${ }^{8,9}$ antibiotic impregnation reduced bacterial colonization both in vitro and in vivo, but the ideal antibiotic has yet to be found. ${ }^{7,10}$ Interesting results were found with the use of triclosan by Chew and associates in vitro, ${ }^{11}$ and Desgrandchamps and colleagues ${ }^{17}$ reported a study in which hydrogel coatings reduced bacterial adhesion, but found a high degree of encrustation when used in vitro.

In previous in vitro and in vivo studies heparinization was found to reduce microbial colonization. ${ }^{12-14}$ There are many hypotheses about how heparin reduces bacterial adhesion; in an environment with a low protein content such as urinary tract, the effect of heparin could result from its hydrophobicity and negative charge. ${ }^{14}$ Ruggieri and associates ${ }^{14}$ showed a $90 \%$ reduction of bacterial adhesion on heparin-coated catheter surfaces, while Hildebrandt and colleagues ${ }^{13}$ demonstrated reductions in stent encrustation by heparin coating in another study. Riedl and co-workers ${ }^{2}$ published an interesting in vivo study, also showing reductions in encrustation in heparin-coated Double $\mathbf{J}$ stents and nephrostomy tubes as determined using electron microscopy.

To our knowledge, our's is the first study in which electron microscopy, elemental, and Micro-IR analysis were used to analyze stent encrustation. Regarding the indications for ureteral

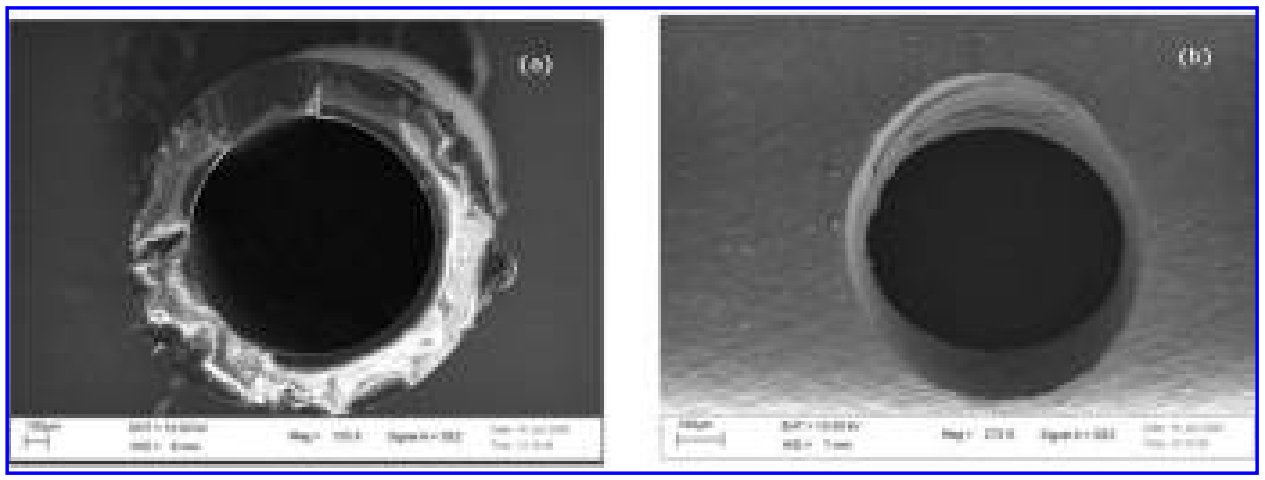

FIG. 6. (a) Cross-section of the heparin-coated stent after being in place for 10 months. No encrustation is visible. (b) Higher magnification of a drainage pore on the outer surface, which is also completely free of encrustation. 


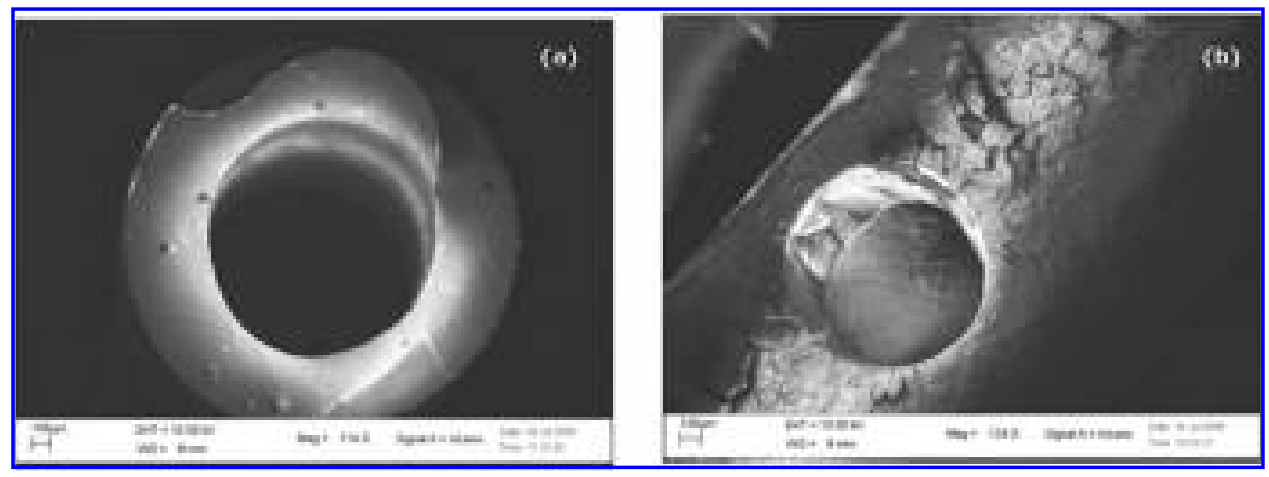

FIG. 7. A heparin-coated stent that was in place for 1 year. (a) Cross-section shows that no encrustations are visible. (b) Here on the external surface there is a bacterial biofilm layer containing sodium chloride and calcium oxide.

stenting, we chose patients with bilateral obstructions to test the coated and uncoated stents in an identical microenvironment. This may be the most important parameter of our study. Riedl and associates ${ }^{2}$ compared two groups of patients, one receiving coated stents and another receiving uncoated stents, but they did not test the two types in the same patient. An important limitation to our study was the small number of patients we tested.

A potential criticism of our study could be the relatively arbitrary nature of how we quantified encrustations, but in our opinion the way we did so, using PC software to perform FESEM analyses, along with the relatively high number of samples, both longitudinal and transverse, helped alleviate this problem.

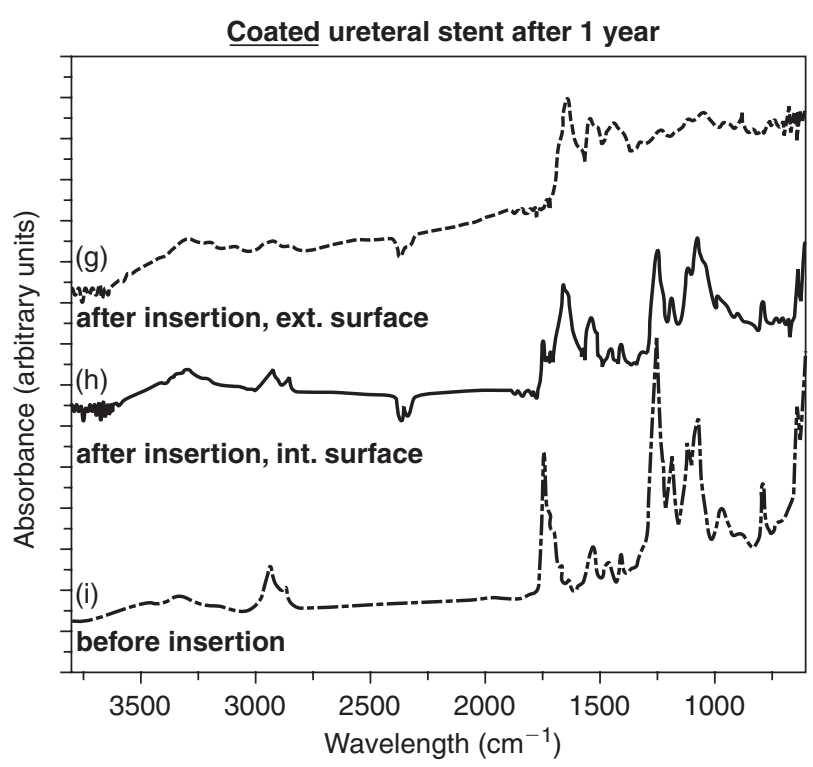

FIG. 8. Micro-IR spectra for the internal and external surfaces of the coated stent after being in place for 1 year. Spectrum (g) was taken at the external surface, showing the presence of bacterial biofilm. Spectrum (h), which was taken at the internal surface, is similar to spectrum (i), which was taken before stent placement; the stent was largely free of encrustations after being in place for 1 year. No changes in the heparin layer or polyurethane were seen.
FESEM, EDS, and Micro-IR results clearly showed the inhibitory role heparin plays in stent encrustation, findings previously suggested by other authors. . $^{2,13,14}$

We found two types of deposits on the stents in our study: (1) amorphous, crystalline inorganic deposits, and (b) bacterial biofilm. Using FESEM and EDS analysis, the encrustations were found to be thicker on the inner surface of the stents than the outer surface, because more urine passes through the lumen of the stent.

Coated Double J stents had smaller encrustations, in terms of both thickness and extension; the heparin coating decreases the incidence of these encrustations, both on the outer and the inner surfaces of the stent. The differences between coated and uncoated stents with regard to their susceptibility to encrustation were clear upon FESEM analysis, and were statistically significant notwithstanding the small number of patients.

The heparin layer can still be seen and analyzed on the surface and on the edges of coated stents, and the heparin did not degrade because the covalent bond guarantees that it will remain stable over time.

Using Micro-IR analysis, we were able to ascertain the organic and inorganic make-up of the encrustation and the substrate. By using infrared spectroscopy in conjunction with microscopy, we were able to accurately map the internal and external surfaces of the stents. Calcium oxalate could easily be detected because of its characteristic spectrum, and was seen on both the coated and uncoated stents; however, our analysis showed that on the coated stents the encrustations were not as uniform and compact as on the uncoated stents. Surprisingly, struvite was not found on EDS and Micro-IR analysis of both the coated and uncoated stents.

We also left heparin-coated stents in place in two patients, one for 10 months and the other for 1 year. These patients had previous problems with obstructed uncoated stents, so we tested the efficacy of coated stents in these problematic patients. After removal, the stents showed no traces of encrustation, and after 10 months the catheter cross-sections were completely free of biofilm, and the drainage pores on the outer surface and the internal lumen, usually sites of heavy encrustation were also free of encrustations. After 1 year in place, the coated stent had biofilm encrustation only on the external surface, and the inner surface was completely free of deposits. Micro-IR analysis confirmed these findings, it clearly showed the chemical bonds be- 
tween the heparin and the surface. As the spectra were nearly identical before insertion and after removal, no change was seen in the heparin layer or the polyethylene substrate after the stent was in place for 1 year. The fact that there was no degradation of the polymer suggests that the polyurethane was unaffected by prolonged contact with urine.

\section{CONCLUSION}

Our data support the hypothesis that heparin reduces stent encrustation after 1 month as well as after 10 and 12 months. Given that no changes were seen in the heparin coating, we can conclude that covalent heparin bonding guarantees a strong bond with the polyurethane surface for long periods of time. In our opinion heparin-coated stents are a good solution for those needing long-term urinary drainage.

\section{REFERENCES}

1. Riedl CR, Plas EG, Hubner WA, Pflueger H. Bacterial colonization of intraureteral stents. Eur Urol 1999;36:53-59.

2. Riedl CR, Witkowski M, Plas E, Pflueger H. Heparin coating reduces encrustation of ureteral stents: A preliminary report. Int J Antimicrob Agents 2002;19:507-512.

3. Gristina AG, Hobgood CD, Webb LX, Myrvik QN. Adhesive colonization of biomaterials and antibiotic resistance. Biomaterials 1987;8:423-426.

4. Stickler D, Ganderton L, King J, Nettleton J, Winters C. Proteus mirabilis biofilm and the encrustation of urethral catheters. Urol Res 1993;21:407-412.

5. Choong S, Wood S, Fry C, Whitfield H. Catheter associated urinary tract infection and encrustation. Int $\mathrm{J}$ Antimicrob Agents 2001;17:305-310.

6. Warren JW, Muncie HL, Hebel JR, Hall-Craggs M. Long-term ureteral catheterization increases risk of chronic pyelonephritis and renal inflammation. J Am Geriatr Soc 1994;42:1286-1290.

7. Raad R, Darouiche J, Dupuis D, et al. Central venous catheters coated with minocycline and rifampin for the prevention of catheter related colonization and bloodstream infections. Ann Intern Med 1997; 127:267-274

8. Riley DK, Classen DC, Stevens LE, Burke JP. A large randomized trial of a silver impregnated urinary catheter: Lack of efficacy and staphylococcal superinfection. Am J Med 1995;98:349-358.
9. Maki DG, Cobb L, Garman JK, Shapiro JM, Ringer M, Helgerson RB. An attachable silver impregnated cuff for prevention of infection with central venous catheter: A prospective randomized multicenter trial. JAMA 1988;127:267-274.

10. Kamal GD, Pfaller MA, Rampe LE. Reduced intravascular catheter infection by antibiotic bonding. JAMA 1991;265:2364-2368.

11. Chew BH, Cadieux PA, Reid G, Densted JD. In vitro activity of triclosan eluting ureteral stent against common bacterial uropathogens. J Endourol 2006;20:949-958.

12. Appelgren P, Ransjo U, Bindslev L, Espersen F, Larm O. Surface heparinization of central venous catheters reduces microbial colonization in vitro and in vivo: Results from a prospective, randomized trial. Crit Care Med 1996;24:1482-1489.

13. Hildebrandt P, Rzany A, Bolz A, Schaldach M. Immobilisiertes heparin als inkrustierungsresistente. Beschichtung auf urologischen implantaten. Biomed Techn 1999;42:123-124.

14. Ruggieri MR, Hanno PM, Levin RM. Reduction of bacterial adherence to catheter surface with heparin. J Urol 1987;138:423-426.

15. Elgue G, Blombaeck M, Olsson P, Reinslfed J. On the mechanism of coagulation inhibition on surfaces with end point immobilized heparin. Thromb Haemost 1993;70:289-293.

16. Kohler-Ockmore J, Feneley RCL. Long term catheterisation of the bladder: Prevalence and morbidity. Br J Urol 1996;77:347-351.

17. Desgrandchamps F, Moulnier F, Daudon M, Teillac P, Leduc A. An in vitro comparison of urease inducted encrustation of Double J stent in human urine. Br J Urol 1997;79:24-27.

Address reprint requests to:

Furio Cauda, M.D.

Dipartimento di Nefrourologia

S.S.C.V.D. per il trattamento integrato della calcolosi urinaria

S.C. Urologia 3

A.S.O. Ospedale Maggiore S. Giovanni Battista Cso Bramante 88, 10125 Turin, Italy

E-mail: furix@ libero.it

\section{ABBREVIATIONS USED}

ATR $=$ attenuated total reflection; EDS $=$ energy dispersive spectroscopy; FESEM = field emission scanning electron microscopy; Micro-IR = micro-infrared spectrophotometry. 